\title{
Comparison of Pulse Propagation and Gain Saturation Characteristics among Different Input Pulse Shapes in Semiconductor Optical Amplifiers
}

\author{
Suchi Barua ${ }^{1}$, Narottam Das ${ }^{1,2}$, Sven Nordholm ${ }^{1}$, and Mohammad Razaghi ${ }^{3}$ \\ ${ }^{1}$ Department of Electrical and Computer Engineering, Curtin University, Perth, WA 6845, Australia \\ ${ }^{2}$ School of Mechanical and Electrical Engineering, University of Southern Queensland, Toowoomba, QLD \\ 4350, Australia \\ ${ }^{3}$ Department of Electrical and Computer Engineering, University of Kurdistan, Sanandaj, Iran \\ *e-mails: suchi.barua@student.curtin.edu.au,narottam.das@usq.edu.au,s.nordholm@curtin.edu.au, and m.razaghi@uok.ac.ir \\ Correspondence: Narottam Das, School of Mechanical and Electrical Engineering, Faculty of Health, \\ Engineering and Sciences, University of Southern Queensland, Toowoomba, West Street, QLD 4350, \\ Australia. Tel: +617 4631 1195; Fax: +617 4631 2526; E-mail: Narottam.Das@usq.edu.au
}

\begin{abstract}
This paper presents the pulse propagation and gain saturation characteristics for different input optical pulse shapes with different energy levels in semiconductor optical amplifiers (SOAs). Finite-difference beam propagation method (FD-BPM) is used to solve the modified nonlinear Schrödinger equation (MNLSE) for the simulation of nonlinear optical pulse propagation and gain saturation characteristics in the SOAs. In this MNLSE, the gain spectrum dynamics, gain saturation are taken into account those are depend on the carrier depletion, carrier heating, spectral hole-burning, group velocity dispersion, self-phase modulation and two photon absorption. From this simulation, we obtained the output waveforms and spectra for different input pulse shapes considering different input energy levels. It has shown that the output pulse shape has changed due to the variation of input parameters, such as input pulse shape, input pulse width, and input pulse energy levels. It also shown clearly that the peak position of the output waveforms are shifted toward the leading edge which is due to the gain saturation of the SOA. We also compared the gain saturation characteristics in the SOA for different input pulse shapes.
\end{abstract}


Keywords: Finite-difference beam propagation method, input pulse shapes, optical pulse propagation, gain saturation, semiconductor optical amplifiers.

\section{Introduction}

In recent years, high-speed communication systems and all-optical signal processing techniques play an important role to avoid electro-optic conversions which may create data-flow bottlenecks. Semiconductor optical amplifiers (SOAs) are widely used in many functional applications, such as wavelength conversion, optical switching, optical signal processing pulse reshaping, and power limiting. SOAs are the key component for short optical pulse amplification and optical switching at a very high speed communications because of their small size, a low switching energy, non-linear characteristics and ability to integrate with other optical devices [1-4].

The purpose of modelling an SOA is to relate the internal variables of the amplifier with external variables, such as the output signal power and output saturation power [3]. When a short input optical pulse is injected into the active region of the SOA, stimulation emission takes place resulting in optical signal amplification. Therefore, the carrier density reduces and causes a drop of the SOA gain [2]. The amplification rate and gain saturation varies according to the input pulse shapes. The modified nonlinear Schrödinger equation (MNLSE) is used in most pulse propagation models that include the SOA nonlinearities [5]. Pulse propagation through an SOA is strongly dependent on the input pulse shape [6].

The main objective of this paper is to investigate the nonlinear optical pulse propagation and gain saturation characteristics depending on different types of input pulse shapes and energy levels in SOAs for high speed communication systems. This analysis is based on the MNLSE considering the non-linearities in SOA, such as selfphase modulation (SPM), two-photon absorption (TPA), group velocity dispersion (GVD), carrier depletion (CD), carrier heating (CH), spectral-hole burning (SHB), gain spectrum dynamics, and gain saturation in the SOA [5, 7]. To solve the MNLSE, finitedifference beam propagation method (FD-BPM) is used because of its short convergence time and excellent accuracy of the simulated results [8-16]. For simulation of pulse propagation with small propagation steps, FD-BPM is considered as the best method 
compared to others [8-14]. In this paper, we have numerically investigated and compared the output waveforms or propagated pulses characteristics and the gain saturation characteristics for different types of input pulse shapes in SOAs. The input pulse shapes were considered as, (i) Secant hyperbolic pulse, (ii) Gaussian pulse, and (iii) Lorentzianshaped pulse.

\section{Modified Nonlinear Schrödinger Equation (MNLSE) for SOA Modelling}

The theoretical model of short optical pulses propagation in SOAs will be briefly described in this section. Starting from Maxwell's equations, we reach to the propagation equation of short optical pulses in SOAs which are governed by the wave equation in the frequency domain [15, 17-22]:

$\nabla^{2} \bar{E}(x, y, z, \omega)+\frac{\varepsilon_{r}}{c^{2}} \omega^{2} \bar{E}(x, y, z, \omega)=0$

where, $\bar{E}(x, y, z, \omega)$ is the electromagnetic field of the pulse in the frequency domain, $c$ is the velocity of light in vacuum and $\varepsilon_{r}$ is the non-linear dielectric constant which is dependent on the electric field in a complex form. By using the slowly varying envelope approximation and integrating the transverse dimensions, the pulse propagation equation in SOAs is [15, 23]:

$\frac{\partial V(\omega, z)}{\partial z}=-i\left\{\frac{\omega}{c}\left[1=\chi_{m}(\omega)+\Gamma \tilde{\chi}(\omega, N)\right]^{1 / 2}-\beta_{0}\right\} V(\omega, z)$

where, $V(\omega, z)$ is the Fourier-transform of $V(t, z)$ representing pulse envelope, $\chi_{m}(\omega)$ is the background (mode and material) susceptibility, $\tilde{\chi}_{m}(\omega)$ is the complex susceptibility which represents the contribution of the active medium, $N$ is the effective population density, $\beta_{0}$ is the propagation constant. The quantity $\Gamma$ represents the overlap/confinement factor of the transverse field distribution of the signal with the active region as defined in [15].

Using mathematical manipulations [19, 23], which includes the real part of the instantaneous non-linear Kerr effect as a single non-linear index $n_{2}$ and by adding the TPA term, the MNLSE for the phenomenological model of semiconductor laser and amplifiers is obtained [24]. 
For this modelling, equation (3) [9-14] is used for the simulation of pulse propagation with different input pulse shapes in SOAs. The MNLSE uses the complex envelope $V(\tau, z)$ function of an optical pulse which is given in equation (3).

$$
\begin{array}{r}
{\left[\frac{\partial}{\partial z}-\frac{i}{2} \beta_{2} \frac{\partial^{2}}{\partial \tau^{2}}+\frac{\gamma}{2}+\left(\frac{\gamma_{2 p}}{2}+i b_{2}\right)|V(\tau, z)|^{2}\right] V(\tau, z)=\left\{\frac{1}{2} g_{N}(\tau)\left[\frac{1}{f(\tau)}+i \alpha_{N}\right]+\frac{1}{2} \Delta g_{T}(\tau)\left(1+i \alpha_{T}\right)\right.} \\
\left.-\left.i \frac{1}{2} \frac{\partial g(\tau, \omega)}{\partial \omega}\right|_{\omega_{0}} \frac{\partial}{\partial \tau}-\left.\frac{1}{4} \frac{\partial^{2} g(\tau, \omega)}{\partial \omega^{2}}\right|_{\omega_{0}} \frac{\partial^{2}}{\partial \tau^{2}}\right\} V(\tau, z)
\end{array}
$$

where,

$$
\begin{aligned}
& g_{N}(\tau)=g_{0} \exp \left(-\frac{1}{W_{s}} \int_{-\infty}^{\tau} e^{-s / \tau_{s}}|V(s)|^{2} d s\right) \\
& f(\tau)=1+\frac{1}{\tau_{s h b} P_{s h b}} \int_{-\infty}^{+\infty} u(s) e^{-s / \tau_{s h b}}|V(\tau-s)|^{2} d s \\
& \Delta g_{T}(\tau)=-h_{1} \int_{-\infty}^{+\infty} u(s) e^{-s / \tau_{c h}}\left(1-e^{-s / \tau_{s h b}}\right)|V(\tau-s)|^{2} d s-h_{2} \int_{-\infty}^{+\infty} u(s) e^{-s / \tau_{c h}}\left(1-e^{-s / \tau_{s h b}}\right)|V(\tau-s)|^{4} d s \\
& \left.\frac{\partial g(\tau, \omega)}{\partial \omega}\right|_{\omega_{0}}=A_{1}+B_{1}\left[g_{0}-g\left(\tau, \omega_{0}\right)\right] \\
& \left.\frac{\partial^{2} g(\tau, \omega)}{\partial \omega^{2}}\right|_{\omega_{0}}=A_{2}+B_{2}\left[g_{0}-g\left(\tau, \omega_{0}\right)\right] \\
& g\left(\tau, \omega_{0}\right)=g_{N}\left(\tau, \omega_{0}\right) / f(\tau)+\Delta g_{T}\left(\tau, \omega_{0}\right)
\end{aligned}
$$

We introduce the frame of the local time $\tau\left(=t-z / v_{g}\right)$ which propagates with the group velocity $v_{g}$ at the centre frequency of an optical pulse. The slowly varying envelope approximation is used in (3), where the temporal variation change of the complex envelope function is very slow compared with the cycle of an optical field. In (3), $V(\tau, z)$ is the time domain complex envelope function of an optical pulse and $|V(\tau, z)|^{2}$ corresponds to the optical intensity or power, and $\beta_{2}$ is the GVD. $\gamma$ is the linear loss, $\gamma_{2 p}$ is the TPA coefficient, $b_{2}\left(=\omega_{0} n_{2} / c A\right)$ is the instantaneous SPM term due to the instantaneous nonlinear refractive index $n_{2}$ (Kerr effect), $\omega_{0}\left(=2 \pi f_{0}\right)$ is the centre angular frequency of the pulse, $c$ is the velocity of light in vacuum, $A(=w d / \Gamma)$ is the effective area ( $d$ and $w$ are the thickness and width of the active region, respectively, and $\Gamma$ is the confinement factor). $g_{N}(\tau)$ is the saturated gain due to CD, $g_{0}$ is the linear gain, $W_{s}$ is the 
saturation energy, $\tau_{s}$ is the carrier lifetime, $f(\tau)$ is the SHB function, $P_{s h b}$ is the SHB saturation power, $\tau_{\text {shb }}$ is the SHB relaxation time, and $\alpha_{N}$ and $\alpha_{T}$ are the line width enhancement factor associated with the gain changes due to the CD and CH. $\Delta g_{T}(\tau)$ is the resulting gain change due to the $\mathrm{CH}$ and TPA. $u(s)$ is the unit step function, $\tau_{c h}$ the $\mathrm{CH}$ relaxation time, $h_{1}$ is the contribution of stimulated emission and free-carrier absorption to the $\mathrm{CH}$ gain reduction, and $h_{2}$ is the contribution of TPA. Finally, $A_{1}$ and $A_{2}$ are the slope and the curvature of the linear gain at $\omega_{0}$ respectively, while $B_{1}$ and $B_{2}$ are constants describing changes in these quantities with saturation. In this simulation, the gain spectrum of an SOA is approximated by the following second-order Taylor expansion in $\Delta \omega$ :

$$
g(\tau, \omega)=g\left(\tau, \omega_{0}\right)+\left.\Delta \omega \frac{\partial g(\tau, \omega)}{\partial \omega}\right|_{\omega_{0}}+\left.\frac{(\Delta \omega)^{2}}{2} \frac{\partial^{2} g(\tau, \omega)}{\partial \omega^{2}}\right|_{\omega_{0}}
$$

The coefficients $\partial g(\tau, \omega) /\left.\partial \omega\right|_{\omega_{0}}$ and $\partial^{2} g(\tau, \omega) /\left.\partial \omega^{2}\right|_{\omega_{0}}$ are related to $A_{1}, A_{2}, B_{1}$ and $B_{2}$ as given by (7) and (8). We assumed the same values of $A_{1}, A_{2}, B_{1}$ and $B_{2}$ as for an AlGaAs/GaAs bulk SOA [9-14].

Generally, the fast Fourier transformation BPM (FFT-BPM) is used for analysis of the optical pulse propagation in optical fibers by successive iterations of the Fourier transformation and the inverse Fourier transformation. In the FFT-BPM, the linear propagation term (GVD term) and phase compensation terms (other than GVD, first- and second- order gain spectrum terms) will be separated in the nonlinear Schrödinger equation for the individual consideration of the time and frequency domain for the optical pulse propagation. However, in our model, (3) includes the dynamic gain change terms, i.e., the first- and second- order gain spectrum terms which are the last two terms of the right side in (3). Therefore, it is not possible to separate (3) into the linear propagation term and phase compensation term, and it is difficult to calculate (3) using the FFT-BPM. For this reason, we have used the FD-BPM [9-14] to solve this MNLSE. 
Table-I: List of the parameters used in simulation [5, 10-14].

\begin{tabular}{|c|c|c|c|}
\hline Parameters & Symbols & Values & Units \\
\hline Length of SOA & $\mathrm{L}$ & 500 & $\mu \mathrm{m}$ \\
\hline Effective area & A & 5 & $\mu m^{2}$ \\
\hline Centre frequency of the pulse & $\mathrm{f}_{0}$ & 349 & $\mathrm{THz}$ \\
\hline Linear gain & $\mathrm{g}_{0}$ & 92 & $\mathrm{~cm}^{-1}$ \\
\hline Group velocity dispersion & $\beta_{2}$ & 0.05 & $\mathrm{ps}^{2} \mathrm{~cm}^{-1}$ \\
\hline Saturation energy & $\mathrm{W}_{\mathrm{s}}$ & 80 & $\mathrm{pJ}$ \\
\hline $\begin{array}{l}\text { Linewidth enhancement factor due to the carrier } \\
\text { depletion }\end{array}$ & $\alpha_{\mathrm{N}}$ & 3.1 & \\
\hline $\begin{array}{l}\text { Linewidth enhancement factor due to the carrier } \\
\text { heating }\end{array}$ & $\alpha_{\mathrm{T}}$ & 2.0 & \\
\hline $\begin{array}{l}\text { The contribution of stimulated emission and free } \\
\text { carrier absorption to the carrier heating gain } \\
\text { reduction }\end{array}$ & $\mathrm{h}_{1}$ & 0.13 & $\mathrm{~cm}^{-1} \mathrm{pJ}^{-1}$ \\
\hline The contribution of two-photon absorption & $\mathrm{h}_{2}$ & 126 & $\mathrm{fscm}^{-1} \mathrm{pJ}^{-2}$ \\
\hline Carrier lifetime & $\tau_{\mathrm{s}}$ & 200 & ps \\
\hline Carrier heating relaxation time & $\tau_{\mathrm{ch}}$ & 700 & fs \\
\hline Spectral-hole burning relaxation time & $\tau_{\mathrm{shb}}$ & 60 & fs \\
\hline Spectral-hole burning saturation power & $\mathrm{P}_{\mathrm{shb}}$ & 28.3 & $\mathrm{~W}$ \\
\hline Linear loss & $\gamma$ & 11.5 & $\mathrm{~cm}^{-1}$ \\
\hline Instantaneous nonlinear Kerr effect & $\mathrm{n}_{2}$ & -0.70 & $\mathrm{~cm}^{2} \mathrm{TW}^{-1}$ \\
\hline Two-photon absorption coefficient & $\gamma_{2 p}$ & 1.1 & $\mathrm{~cm}^{-1} \mathrm{~W}^{-1}$ \\
\hline $\begin{array}{l}\text { Parameter describing second-order Taylor } \\
\text { expansion of the dynamically gain spectrum. }\end{array}$ & $\begin{array}{l}\mathrm{A}_{1} \\
\mathrm{~B}_{1} \\
\mathrm{~A}_{2} \\
\mathrm{~B}_{2}\end{array}$ & $\begin{array}{l}0.15 \\
-80 \\
-60 \\
0\end{array}$ & $\begin{array}{l}\text { fs } \mu \mathrm{m}^{-1} \\
\text { fs } \\
\mathrm{fs}^{2} \mu \mathrm{m}^{-1} \\
\mathrm{fs}^{2}\end{array}$ \\
\hline
\end{tabular}

\section{Simulation Results and Discussion}

In this section, simulation results of single pulse propagation and gain saturation characteristics are discussed and compared for different input pulse shapes in SOAs. The 
parameters of a bulk SOA (AlGaAs/GaAs, double heterostructure) is used for the simulation as listed in Table-I [5, 10-14]. The length of the SOA is $500-\mu \mathrm{m}$. We have obtained all the results with a propagation step $\Delta z$ of $10-\mu \mathrm{m}$.

\subsection{Optical Pulse Propagation Characteristics for Different Input Pulse Shapes}

Optical pulse propagation in SOAs has attracted much more attention due to its potential applications in high-speed optical communication systems, such as wavelength converter and switching. There are several applications in all optical signal processing, such as clock recovery and optical time division demultiplexing, where the SOAs are used to amplify high energy optical pulses with the pulse width of the order of picoseconds [6].

In this subsection, optical pulse propagation characteristics for different input pulse shapes in SOAs are discussed. For the simulation of optical pulse propagation characteristics, different types of input pulse shapes are considered for the simulation in SOAs, such as (i) Secant hyperbolic pulse, (ii) Gaussian pulse, and (iii) Lorentzianshaped pulse). The mathematical formulations of different input pulse shapes in time domain are as follows:

(i) Secant hyperbolic pulse shape:

$$
P(t)=\sqrt{\left(E_{\text {in }} / 2 t_{0}\right)} \operatorname{sech}\left(t / t_{0}\right)
$$

(ii) Gaussian pulse shape:

$$
P(t)=\sqrt{\left(E_{\text {in }} / t_{0} \sqrt{\pi}\right)} \exp \left(-t^{2} / 2 t_{0}{ }^{2}\right)
$$

(iii) Lorentzian pulse shape:

$$
P(t)=\sqrt{\left(2 E_{\text {in }} / t_{0} \pi\right)}\left(1+t^{2} / t_{0}^{2}\right)^{-1}
$$

where, $E_{\text {in }}$ is the input pulse energy in $\mathrm{pJ}, t$ is time in $\mathrm{s}$, and $t_{0}$ is the parameter that controls the width of the input pulse in s.

Figure 1 illustrates the simulation model for the propagation of optical pulses in an SOA. An optical pulse is injected into the input facet of the SOA, where the input pulse position is at $z=0$. The pulse propagated over the SOA length $500-\mu \mathrm{m} \mathrm{SOA}$. 
Figure 2 shows the simulation results of optical pulse propagation characteristics in an SOA, when the input pulse width is 10 ps. Output waveforms for Secant hyperbolic pulse shape, (Gaussian pulse shape and Lorentzian pulse shape are observed in figure 2(i), when the input pulse energies are (a) 2 pJ, (b) 1 pJ and (c) 500 fJ. The considered sampling time step $(\Delta t)$ is 0.025 ps. The output pulse energy increases with the increase of input pulse energy level. It is clearly observed that higher output pulse energy is achieved by Gaussian pulse and lower output pulse energy is achieved by Lorentzian pulse for all considered input pulse energies (high to low). The calculated peak output powers are 4.2693 W, 4.5268 W, and 4.0091 W for Secant hyperbolic, Gaussian and Lorentzian pulses, respectively when input energy is $2 \mathrm{pJ}$. The calculated peak output powers are 2.89 W, 3.06 W and 2.71 W for Secant hyperbolic, Gaussian and Lorentzian pulses, respectively when input energy is $1 \mathrm{pJ}$. When input energy is low (such as $500 \mathrm{fJ}$ ), the calculated peak output powers are $1.77 \mathrm{~W}, 1.88 \mathrm{~W}$, and $1.66 \mathrm{~W}$ for Secant hyperbolic, Gaussian and Lorentzian pulses, respectively. From this calculation it can be concluded that, higher output energy can be achieved for higher input energy for all those three pulse shapes and vice versa. As the input pulse width is very shorter than the considered carrier lifetime, the leading edge of the pulse saturates the amplifier and the trailing edge experiences a lower gain, so the output pulse shape becomes asymmetric. Comparing all three pulse shapes with respect to different input pulse energy levels, it can be observed that, the output pulse shapes become more asymmetric for higher input energy and less asymmetric for lower input energy.

Figure 2(ii) shows the frequency spectra of propagated output pulses for Secant hyperbolic pulse shape, Gaussian pulse shape and Lorentzian pulse shape when the input pulse energies are (a) 2 pJ, (b) 1 pJ and (c) 500 fJ. Frequency spectra were obtained by performing the FFT on the temporal pulse shapes as shown in Fig. 2 (i). Output spectral shape shifting is observed toward the lower frequency side, which is due to the gain saturation of the SOA and SPM effects. For weak input pulse energies (i.e., $\leq 500 \mathrm{fJ}$ ), none of the output frequency spectra has been shifted toward the lower frequency side. It can be clearly seen from the figure that the amount of frequency shift is $\sim-8.3 \mathrm{GHz}$ for Secant hyperbolic and Gaussian pulses when the input pulse energy is $2 \mathrm{pJ}$ and $1 \mathrm{pJ}$. 
From the simulated results, it has confirmed that there is no red shifting occurs for Lorentzian pulse for the considered input pulse energies (i.e., $500 \mathrm{fJ} \sim 2 \mathrm{pJ}$ ). From this observation it can be said that, red shifting has been occurred for higher input pulse energy levels (i.e., 1 pJ 2 pJ) for Secant hyperbolic and Gaussian pulse shapes. A wider spectral broadening is observed for Gaussian pulse when input energy $2 \mathrm{pJ}$. Besides that some oscillatory structures (i.e., dips) are observed in the upper frequency side of the frequency spectra, which is due to the SPM effects $[9,10,15]$. The physical mechanism behind the spectral shift and distortion is the SPM, occurring as a result of index nonlinearities induced by the gain saturation [9-16].

\subsection{Gain Saturation Characteristics for Different Input Pulse Shapes}

In this subsection, gain saturation characteristics for different input pulse shapes in SOAs are discussed. Fig. 3 shows the saturated gain versus the output pulse (waveform) energy characteristics for the input pulse shapes: (a) Secant hyperbolic, (b) Gaussian, and (c) Lorentzian. These pulse shapes are Fourier transform limited. The FWHM of the input pulses are varied from 0.5 ps to 10 ps. The saturation behaviour is different for short (such as, $<1 \mathrm{ps}$ ) and long (such as, $>1 \mathrm{ps}$ ) pulses. With the low input pulse energies (such as, $\sim 0.1 \mathrm{pJ}$ ), the gain is unsaturated (i.e., a linear gain). Also, when the input pulse duration is short (i.e., $<1$ ps) then the gain saturates at low output energies and it is true for all the three pulse shapes. It is observed clearly that the gain saturation is pulsewidth dependent and output saturation energy increases with the increase of pulsewidth. Comparing among the three pulse shapes, it is observed clearly that gain saturates at higher output energy for the Lorentzian input pulse shape with particular input pulse energy and achieve higher gain for all pulse widths.

Fig. 4 shows comparison of the gain saturation among the different input pulse shapes, when (a) FWHM $=0.5$ ps, (b) FWHM $=3$ ps and (c) FWHM $=10$ ps. For shorter pulse width (such as, $\sim 0.5 \mathrm{ps}$ ), gain saturates at lower output energy for all three pulse shapes. From this observation, it can be said that, the output saturation energy is dependent on the input pulse width. Output saturation energy increases with the increase of input pulse width and decreases with the decrease of input pulse width (shorter pulse). Also the linear gain is very similar for all the pulse shapes when the input pulse energy is low (such as, 
$\sim 0.5 \mathrm{pJ}$ ). From the figures, we can see that, Gaussian pulse reaches to saturation quicker (green solid line) than the other two pulses. It can be also observed that, with the increase of pulse duration (i.e., pulsewidth), the gain saturation characteristics are becoming similar for Secant hyperbolic and Gaussian pulse shapes.

When the pulse width is very short i.e., $\sim 0.5$ ps (as shown in Fig. 4(a)), the gain saturates at lower output pulse energies for all three input pulse shapes (i.e., low input pulse energies at the linear region) but the output saturation energy varies significantly for different input pulse shapes (i.e., higher input pulse energies at the nonlinear region). Hence, it is noted that the variation of output pulse energy is not much significant for low input pulse energies as it is in the linear region. However, the variation of output pulse energy varies significantly for higher input pulse energies as it is in the nonlinear region. These effects are clearly shown in Fig. 4(a) for different short input pulse shapes. In addition, when the input pulse widths increased to 3-ps and 10-ps (i.e., Figs. 4(b) and 4(c)), then the output pulse energy variation is not significant for different input pulse shapes even for higher input pulse energies at the nonlinear region. These effects are clearly shown in Fig. 4(b) and 4(c) for different input pulse shapes. Therefore, for short input pulses i.e., $0.5 \mathrm{ps}$, it is clear that three identical lines for three different input pulse shapes (i.e., similar trend but not overlapped).

However, with the increase of input FWHM (such as, 3 ps $~ 10$ ps), the gain saturation characteristics is becoming very similar (i.e., mostly overlapped) for Secant hyperbolic and Gaussian pulses. That means with the increase of pulsewidths (>1 ps), the gain saturation characteristics has become more similar for Secant hyperbolic and Gaussian

pulse. For all considered FWHM, higher gain saturation i.e., higher output energy has been obtained for Lorentzian pulse shape.

\section{Conclusion}

In conclusion, optical pulse propagation and gain saturation characteristics in an SOA are analyzed for different input pulse shapes. It can be observed from the output waveforms that the peak positions are shifted toward the leading edge due to the gain saturation of the SOA and higher output power has been calculated for Gaussian pulse at all considered input energies. Red shifting is occurred with higher input energy $(1 \mathrm{pJ}-2 \mathrm{pJ})$ 
for Secant hyperbolic and Gaussian pulses but not for Lorentzian pulse. That means the gain is not saturated (linear gain) for Lorentzian pulse at $2 \mathrm{pJ}$ input energy. Lorentzian pulse requires more input energy than other two pulses to reach in saturation. However, for lower input energy (such as $500 \mathrm{fJ}$ ), no red shifting is occurred for any of the three pulses. Several dips can be observed at the higher frequency side of the output spectra due to the SPM effect. Also it can be observed that the highest output pulse energy was obtained by the Lorentzian pulse shapes. At low input pulse energies, the gain is unsaturated but with increasing of input pulse energies, the gain reaches to the saturation. The saturated output pulse energy increases with increasing of pulsewidth for all considered pulse shapes. The Gaussian pulse shape is reaching to the gain saturation faster compared to the Secant hyperbolic and Lorentzian pulses. Moreover, the gain saturation characteristics for Secant hyperbolic and Gaussian pulses are very similar when the pulsewidth is $>1$ ps.

\section{ACKNOWLEDGEMENT}

This research is supported by the Faculty of Science and Enginering, Curtin University, Perth, WA, Australia and Curtin University Postgraduate Student Association (CUPSA) for providing the postgraduate student travel support to attend the International Conferences in Spain and Singapore for paper presentation.

\section{References}

[1] P. P. Baveja, A. M. Kaplan, D. N. Maywar, G. P. Agrawal, "Pulse amplification in semiconductor optical amplifiers with ultrafast gain-recovery times," in proc. of the SPIE 7598, Optical Components and Materials VII, vol. 7598, pp. 2-11, February, 2010.

[2] A. A. E. Aziz, W. P. Ng, Z. Ghassemlooy, M. H. Aly, and M. F. Chiang, "Optimisation of the key SOA parameters for amplification and switching," in proc. of the 9th Annual Postgraduate Symposium on the Convergence of Telecommunications, Networking and Broadcasting, PGNET, Liverpool, pp. 107111, 2008. 
[3] A. A. Shalaby, "Characterisation and optimisation of the semiconductor optical amplifier for ultra-high speed performance,” Doctoral Dissertation, Northumbria University, Newcastle, UK, 2012.

[4] X. Yang, Q. Weng, and W. Hu, "High-speed all-optical switches based on cascaded SOAs," Selected Topics on Optical Amplifiers in Present Scenario, Dr Sisir Garai (Ed.), InTech, pp. 25-46, 2012.

[5] M. Y. Hong, Y. H. Chang, A. Dienes, J. P. Heritage, and P. J. Delfyett, "Subpicosecond pulse amplification in semiconductor laser amplifiers: Theory and experiment,” IEEE J. Quantum Electron., vol. 30, pp. 1122-1131, 1994.

[6] M. J. Connelly, L. P. Barry, B. F. Kennedy, D. A. Reid, "Numerical analysis of picoseconds pulse propagation in a tensile-strained semiconductor optical amplifier with parameter extraction using frequency resolved optical gating," Optical and quantum electronics, vol. 40, Issue. 5-6, pp. 411-418, April 2008.

[7] Y. Said and H. Rezig "SOAs nonlinearities and their applications for next generation of optical networks," Advances in Optical Amplifiers, Edited by Prof. Paul Urquhart, InTech, pp. 27-52, 2011.

[8] S. D. Conte and Carl de Boor, Elementary Numerical Analysis: An Algorithmic Approach, Third Edition, McGraw-Hill Book Company Co., Singapore, 1980.

[9] M. Razaghi, V. Ahmadi, and M. J. Connelly, "Comprehensive finite-difference time-dependent beam propagation model of counter-propagating picosecond pulses in a semiconductor optical amplifier," IEEE/OSA Journal of Lightwave Tech., vol. 27, No. 15, pp. 3162-3174, August 2009.

[10] N. K. Das, Y. Yamayoshi, and H. Kawaguchi, "Analysis of basic four wave mixing characteristics in a semiconductor optical amplifier by the finite-difference beam propagation method,” IEEE Journal of Quantum Electronics, vol. 36, No. 10, pp. 1184-1192, October 2000.

[11] N. K. Das and N. C. Karmakar, "Nonlinear propagation and wave mixing characteristics of pulses in Semiconductor optical amplifiers," Microwave and Optical Tech. letters, vol. 50, No. 5, pp. 1223-1227, May 2008.

[12] S. R. Hosseini, M. Razaghi, and N. K. Das, "Analysis of ultrafast nonlinear phenomena's influences on output optical pulses and four-wave mixing 
characteristics in semiconductor optical amplifiers,” Opt. Quantum Electron, vol. 42(11-13), pp. 729-737, April 2011.

[13] S. R. Hosseini, M. Razaghi, and N. K. Das, “Analysis of non-linear refractive index influences on four-wave mixing conversion efficiency in semiconductor optical amplifiers,” Opt. Laser Tech., vol. 44(3), pp. 528-533, April 2012.

[14] N. K. Das, M. Razaghi, and S. R. Hosseini, "Four-wave mixing in semiconductor optical amplifiers for high speed communication,” in proc. of the $5^{\text {th }}$ International conference on Computers and Devices for Communication 2012 (CODEC2012), Kolkata, India Dec. 16-19, 2012.

[15] G. P. Agrawal and N. A. Olsson, "Self-phase modulation and spectral broadening of optical pulses in semiconductor laser amplifier,” IEEE Journal of Quantum Electronics, vol. 25, No. 11, pp. 2297-2306, November 1989.

[16] P. Borri, S. Scaffetti, J. Mørk, W. Langbein, J. M. Hvam, A. Mecozzi, and F. Martelli, "Measurement and calculation of the critical pulsewidth for gain saturation in semiconductor optical amplifiers,” Optics Communications, vol. 164, pp. 51-55, June 1999.

[17] G. P. Agrawal, “Nonlinear Fiber Optics”, Academic Press, Calif., San Diego, 1989.

[18] A. Yariv, "Optical Electronics," 4th Edition, Saunders College Publishing, San Diego, 1991.

[19] E. G. Sauter, “Nonlinear Optics,” John Wiley \& Sons, Inc. New York, 1996.

[20] S. Kai, M. Premaratne, "Effects of SPM, XPM, and four-wave-mixing in L-band EDFAs on fiber-optic signal transmission,” IEEE Photonics Technology Letters, vol. 12, no. 12, pp. 1630-1632, Dec. 2000.

[21] C. Dissanayake, M. Premaratne, I. Rukhlenko, and G. Agrawal, "FDTD modeling of anisotropic nonlinear optical phenomena in silicon waveguides,” Optics Express, vol. 18, pp. 21427-21448, 2010.

[22] M. Premaratne and G. P. Agrawal, "Light Propagation in Gain Media: Optical Amplifiers,” Cambridge University Press, 2011.

[23] A. Dienes, J. P. Heritage, C. Jasti, and M. Y. Hong, "Femtosecond optical pulse amplification in saturated media,” J. Opt. Soc. Am. B, vol. 13, pp. 725-734, 1996. 
[24] M. Y. Hong, Y. H. Chang, A. Dienes, J. P. Heritage, P. J. Delfyett, Sol Dijaili, and F. G. Patterson "Femtosecond self- and cross-phase modulation in semiconductor laser amplifiers,” IEEE Journal of Selected Topics in Quantum Electronics, vol. 2, no. 3, pp. 523-539, 1996.

Fig. 1. Schematic diagram for the simulation of nonlinear pulse propagation in SOAs. Here, $|V(\tau, 0)|^{2}$ and $|V(\tau, z)|^{2}$ are the input and output (after propagating a distance $z$ ) pulses power or intensity of the SOA.

Fig. 2 (i). Output waveforms for Secant hyperbolic pulse, Gaussian pulse and Lorentzian pulse when input pulse energy levels are (a) 2 pJ, (b) 1 pJ and (c) $500 \mathrm{fJ}$.

Fig. 2 (ii). Output spectra for Secant hyperbolic pulse, Gaussian pulse and Lorentzian pulses when input pulse energy levels are (a) 2 pJ, (b) 1 pJ and (c) 500 fJ.

Fig. 3. Gain saturation characteristics for different input pulse shapes with different FWHM, such as 0.5-ps to 10-ps. The input pulse shapes are: (a) Secant hyperbolic pulse, (b) Gaussian pulse, and (c) Lorentzian pulse.

Fig. 4. Comparison of gain saturation characteristics for different types of input pulse shapes, when (a) FWHM = 0.5 ps, (b) FWHM $=3$ ps and (c) FWHM $=10$ ps.

Fig. 1.

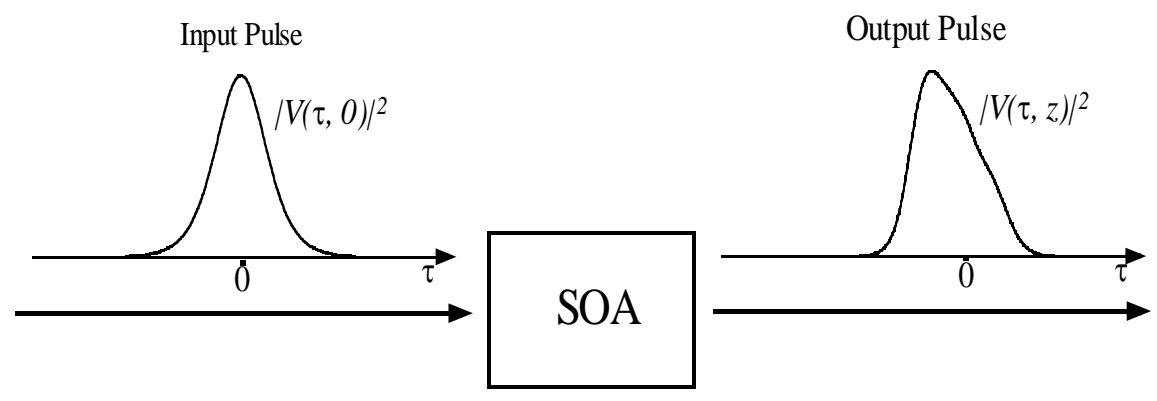


Fig. 2 (i).

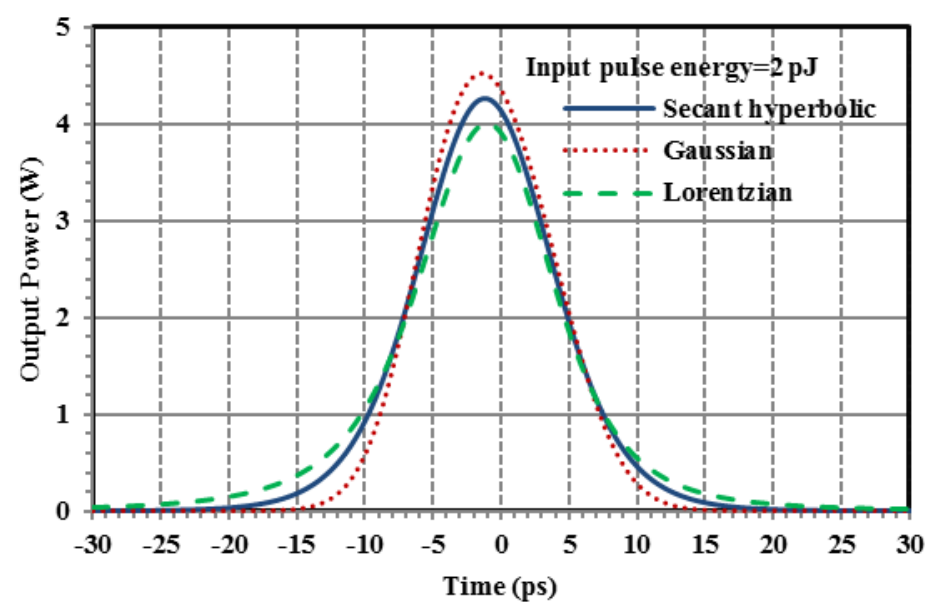

(a)

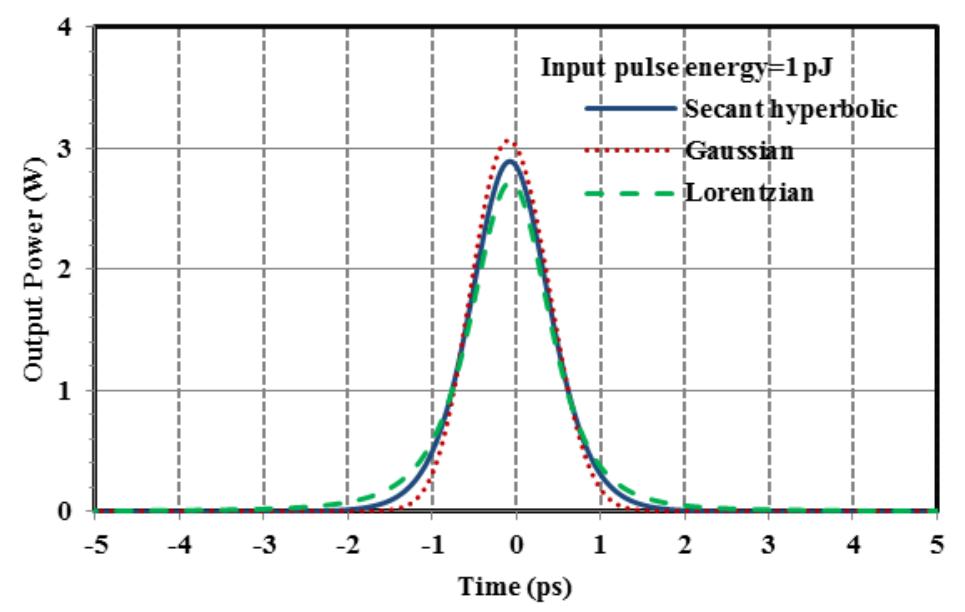

(b)

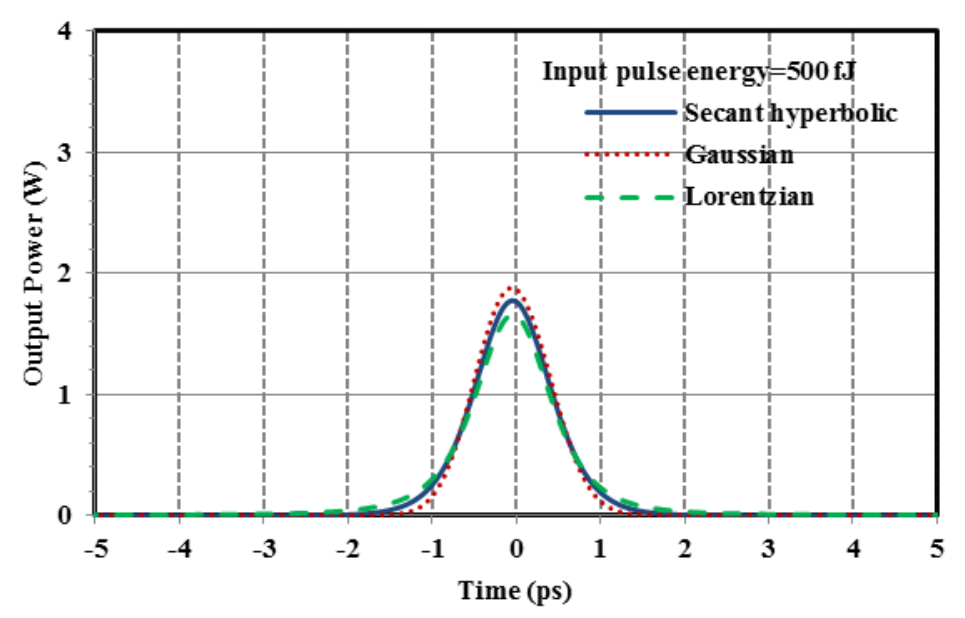

(c) 
Fig. 2 (ii).

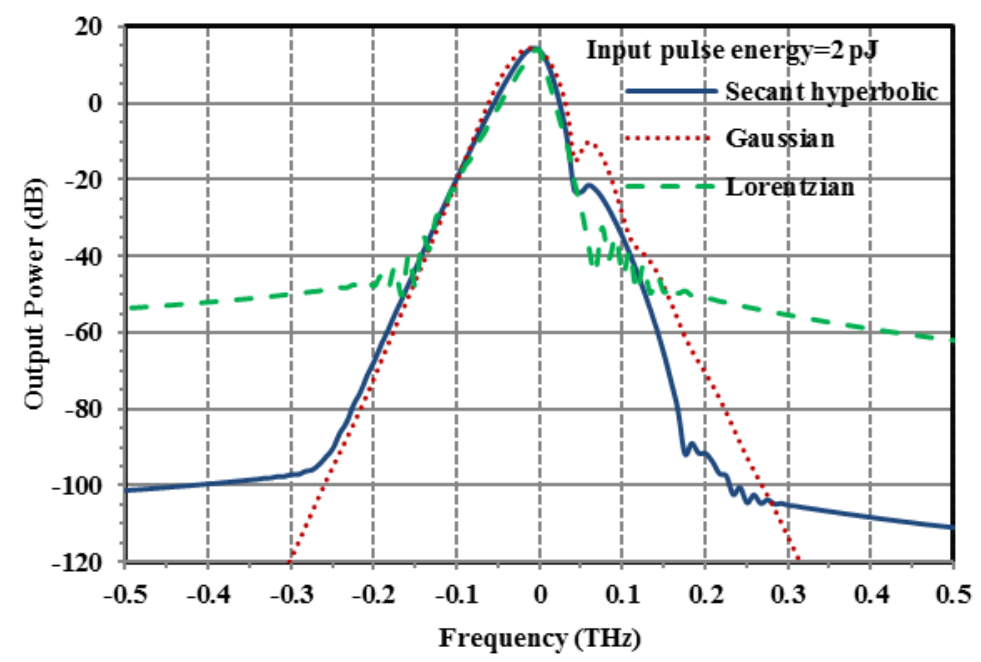

(a)

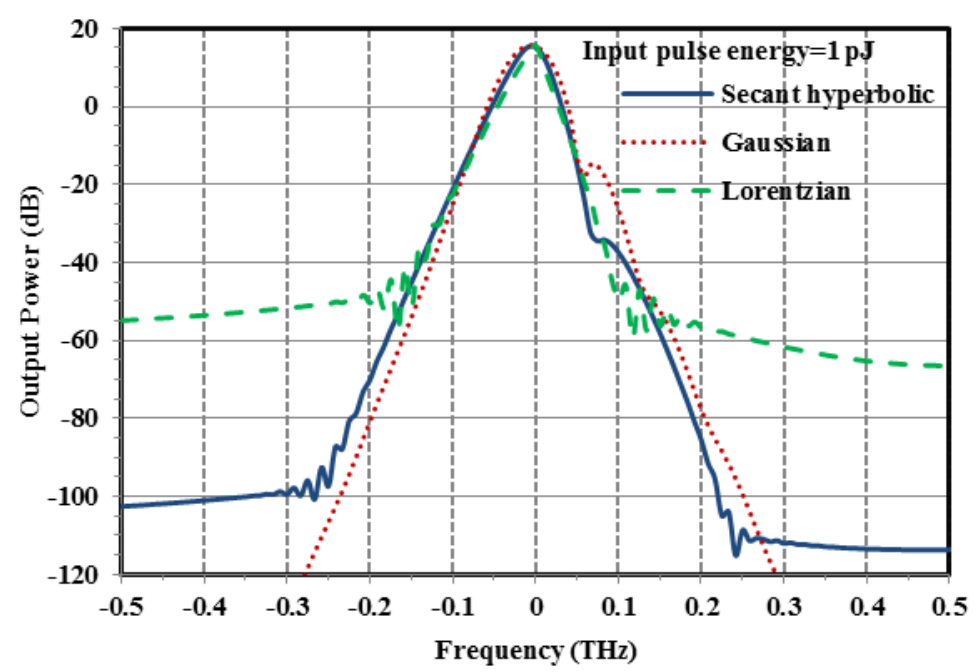

(b)

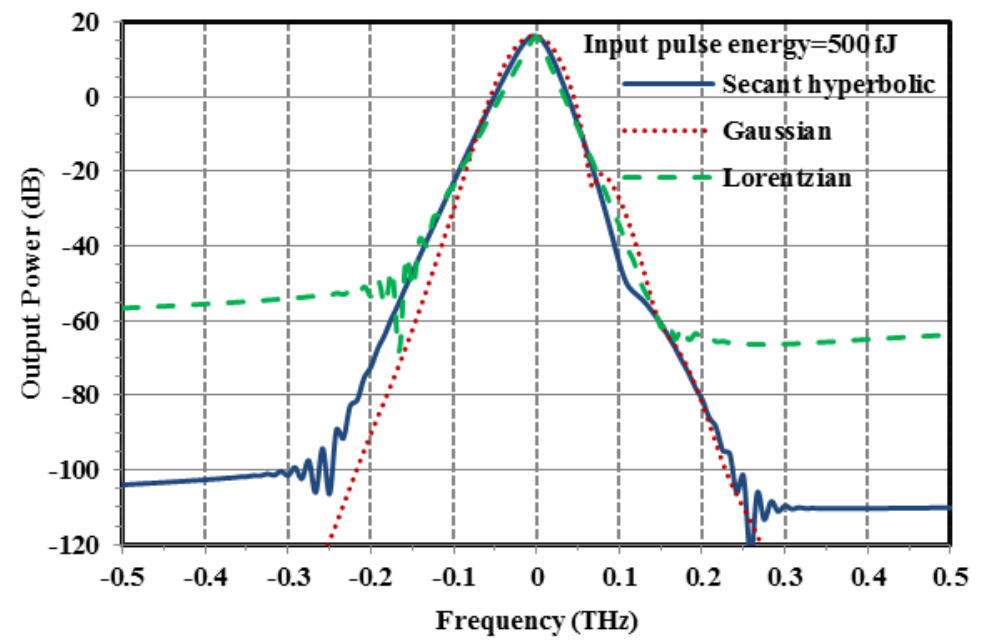

(c) 
Fig. 3.

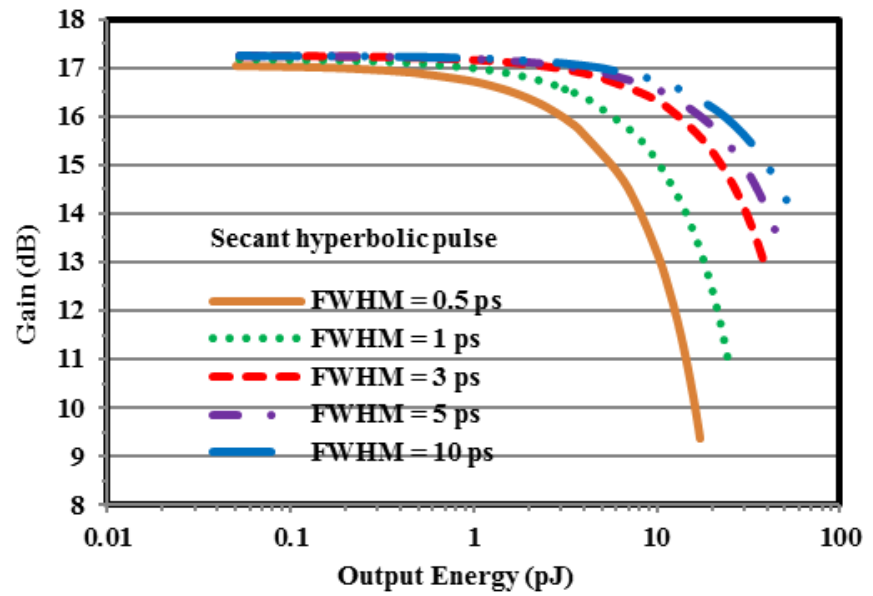

(a)

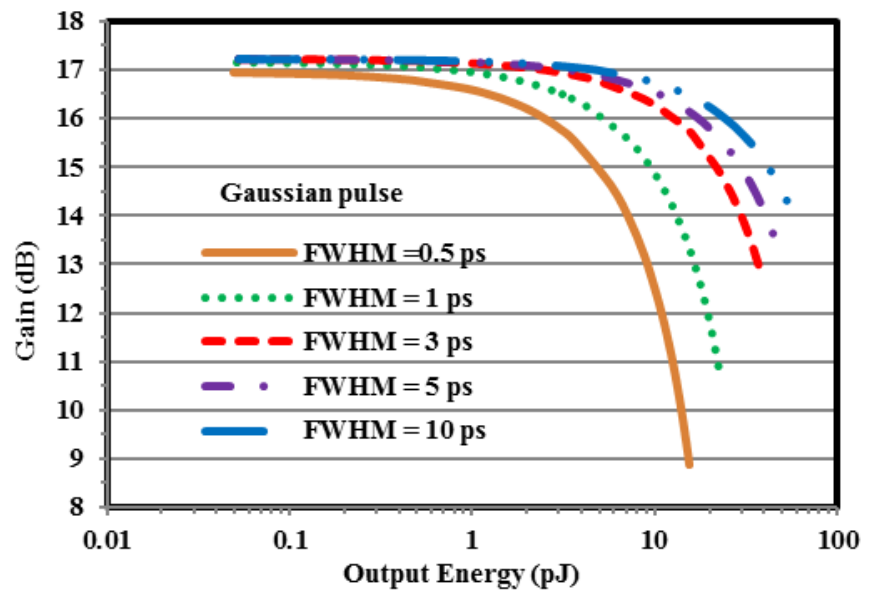

(b)

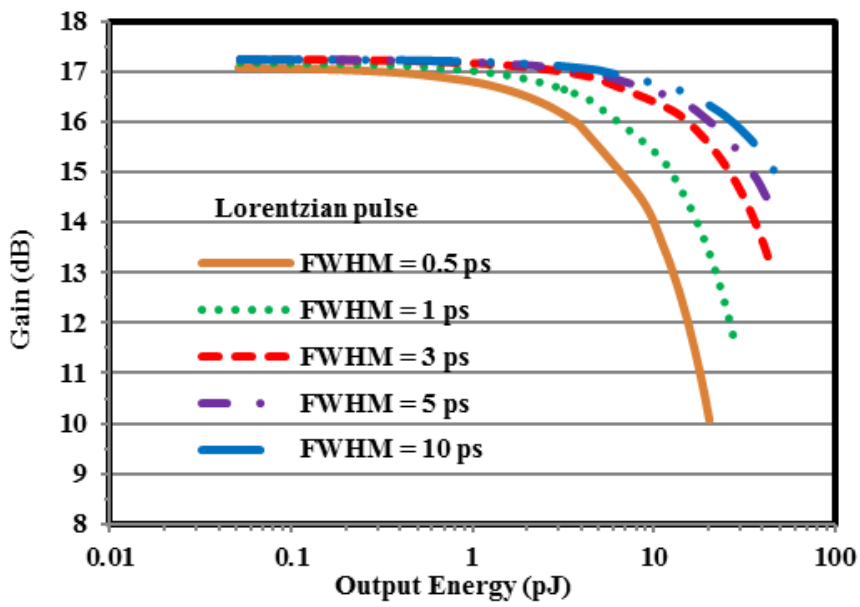

(c) 
Fig. 4.

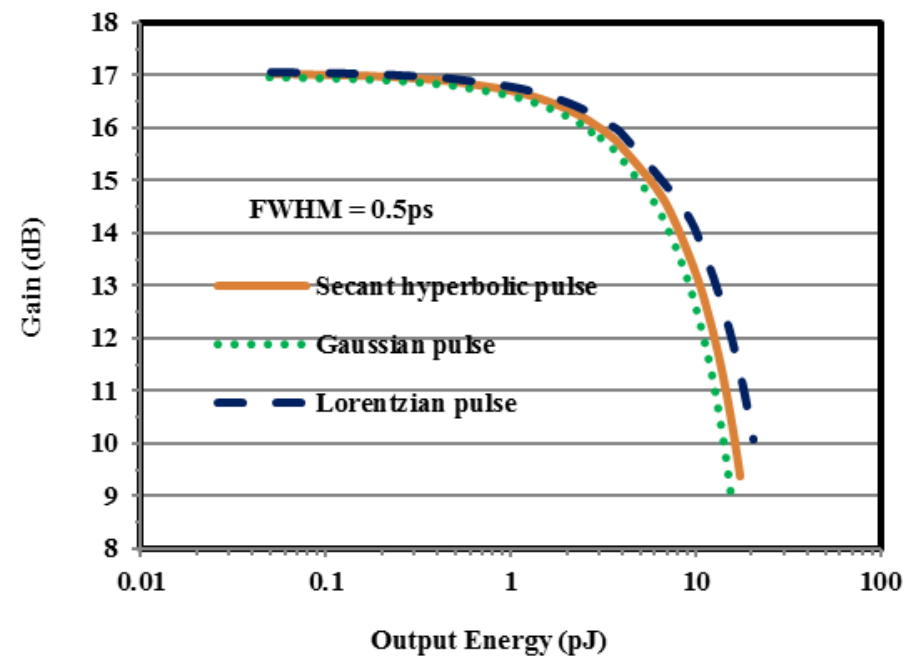

(a)

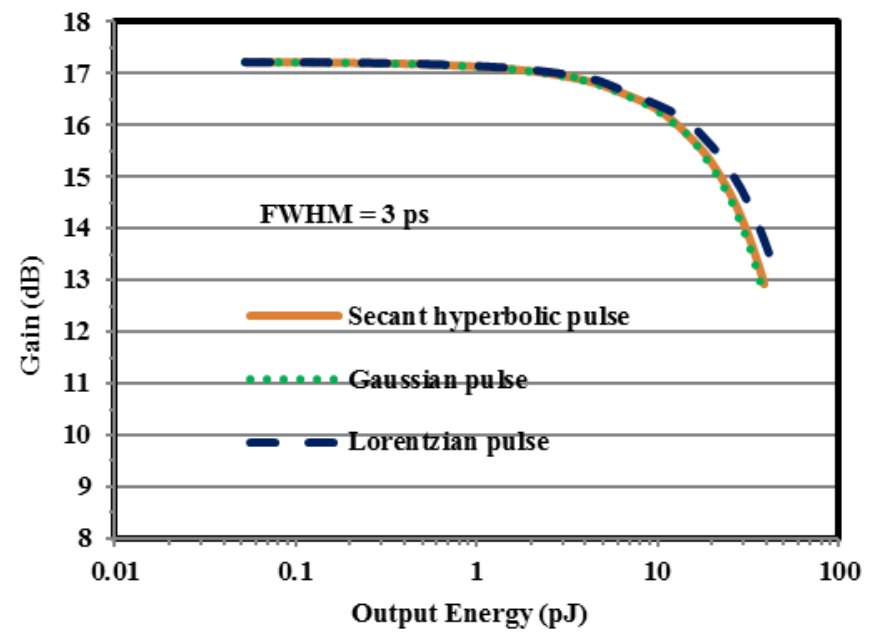

(b)

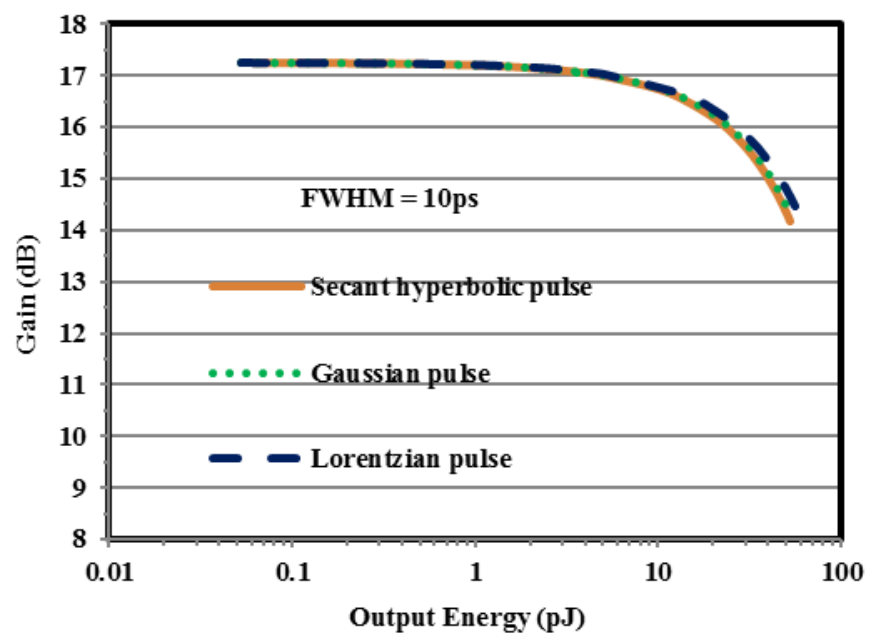

(c) 